Juan Garrido, Francisco Vázquez, Fernando Morilla and Julio E. Normey-Rico, Smith Predictor with Inverted Decoupling for Square Multivariable Time Delay Systems, International Journal of Systems Science, volume 47, number 2, pp. 374-388, 2016,

ISSN 0020-7721, The Version of Record of this manuscript has been published and is available in http://www.tandfonline.com/doi/abs/10.1080/00207721.2015.1067338 (http://dx.doi.org/10.1080/0020772 1.2015.1067338.)

\title{
SMITH PREDICTOR WITH INVERTED DECOUPLING FOR SQUARE MULTIVARIABLE TIME DELAY SYSTEMS
}

\author{
Juan Garrido ${ }^{\mathrm{a}}$, Francisco Vázquez ${ }^{\mathrm{a}}$, Fernando Morilla ${ }^{\mathrm{b}}$, Julio E. Normey-Rico ${ }^{\mathrm{c}}$ \\ ${ }^{a}$ Dept. of Computer Science and Numerical Analysis, University of Cordoba, Campus de \\ Rabanales, 14071, Cordoba, Spain
}

Fax: (+34)957218729; e-mail: juan.garrido@uco.es,fvazquez@uco.es

${ }^{b}$ Dept. of Computer Science and Automatic Control, UNED, Juan del Rosal 16, 28040, Madrid,Spain,fmorilla@dia.uned.es

${ }^{c}$ Dept. de Automação e Sistemas, Universidade Federal de Santa Catarina, 88040-900, Florianópolis, SC, Brazil, julio.normey@ufsc.br

\begin{abstract}
This paper presents a new methodology to design multivariable Smith predictor for $n \times n$ processes with multiple time delays based on the centralized inverted decoupling structure. The controller elements are calculated in order to achieve good reference tracking and decoupling response. Independently of the system size, very simple general expressions for the controller elements are obtained. The realizability conditions are provided and the particular case of processes with all of its elements as first order plus time delay systems is discussed in more detail. A diagonal filter is added to the proposed control structure in order to improve the disturbance rejection without modifying the nominal set-point response and to obtain a stable output prediction in unstable plants. The effectiveness of the method is illustrated through different simulation examples in comparison with other works.
\end{abstract}

Keywords: Smith predictor; multivariable time delay systems; decoupling control. 


\section{Introduction}

Time delays arise in many industrial processes as a consequence of different phenomena such as transport times of mass, information or energy; accumulation of time lags in processes interconnected in series; or processing time (Normey-Rico \& Camacho, 2007). Time delays affect the performance of traditional control systems because they can lead to very poor system response as they prevent high controller gain from be used in order to avoid instability. The Smith Predictor (SP) was the first compensator specially designed for singleinput single output (SISO) systems with time delay (Smith, 1957). It allows the elimination of the time delay in the characteristic equation and gives an output prediction for set point changes. In the last years, different approaches of the SP have been developed to overcome some drawbacks of its initial proposal and to improve its performance (Normey \& Camacho, 2008; Normey-Rico \& Camacho, 2006; Palmor, 1996). Other important aspects such as compensation of systems with time-varying delay (Krstic, 2010) and control of systems with uncertain time delay (Bresch-Pietri, Chauvin, \& Petit, 2012; Zhang, Shi, \& Wang, 2014; Zhang \& Wang, 2015) have been addressed recently.

On the other hand, most industrial processes are multiple-inputs multiple-outputs (MIMO) systems, which are much more difficult to control compared with SISO counterparts because of the existence of interactions between the measurement signals and the control signals. In presence of time delays, the control system design becomes even more difficult because each output is affected by each input with different time delays (Jerome \& Ray, 1986). As a result, a transfer function matrix representation of the MIMO process is preferred in these cases (Wang, Zhang, \& Chiu, 2002). Different approaches have been developed in order to design controller for multivariable systems with multiple time delays. Some authors have developed directly multivariable methodologies based on the conventional unity feedback structure: decoupling control (Liu, Zhang, \& Gao, 2007; Morilla, Garrido, \& 
Vazquez, 2013; Wang, Zhang, \& Chiu, 2003; Xie, Shiehb, Pana, Tsaic, \& Canelond, 2014), multivariable PID controllers (Garrido, Vázquez, \& Morilla, 2014b; Vijay-Kumar, Rao, \& Chidambaram, 2012; Xiong, Cai, \& He, 2007) or $\mathrm{H}_{\infty}$ controllers (Galdos, Karimi, \& Longchamp, 2010; Mirkin, Palmor, \& Shneiderman, 2011). The main disadvantage of these methods is the complexity of the control design procedure that increases with the size of the process. In addition, the resultant controller elements need to be generally reduced for implementation. With decentralized controllers (Huang, Jeng, Chiang, \& Pan, 2003; Vázquez, Morilla, \& Dormido, 1999), the resultant control system is simpler; however, important interactions can arise and produce a poor closed-loop performance.

Other authors propose more advanced structures such as multivariable IMC schemes (Garrido, Vázquez, \& Morilla, 2014a; He, Cai, \& Wu, 2006; Wang et al., 2002), or extensions of the SP to the multivariable case using a similar scheme to that of Figure 1, where G(s) is the plant, $G_{n}(s)$ is the nominal model of the plant, $G_{0}(s)$ is the fast model of the process and $\mathrm{C}(\mathrm{s})$ is the primary controller.

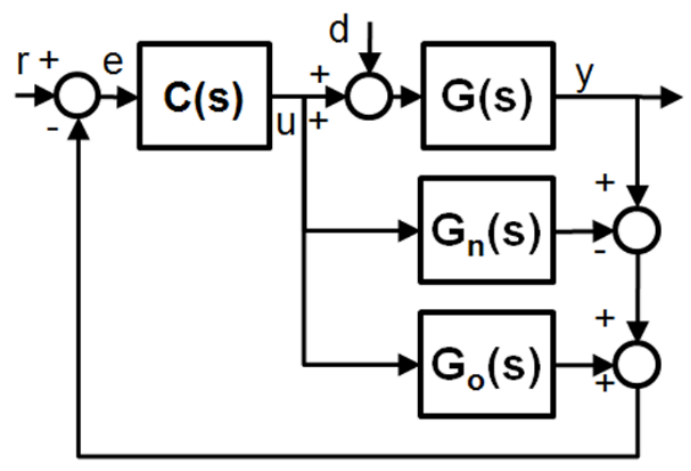

Figure 1. Smith Predictor scheme.

In order to apply SP to multivariable systems, two approaches can be usually found. The first one consists in designing a decoupling compensator $D(\mathrm{~s})$ for the original process $G(\mathrm{~s})$ in order to obtain a diagonal (or diagonal dominant) apparent process, and then, applying the SP to this apparent process $\mathrm{H}(\mathrm{s})=\mathrm{G}(\mathrm{s}) \cdot \mathrm{D}(\mathrm{s})$ (Figure 2) (Wang, Zou, \& Zhang, 2000). Then, the SP design can be carried out as that of SISO case. The second one and more 
common applies simultaneously multivariable control and SP (Rao \& Chindambaram, 2006) using the scheme of Figure 1.

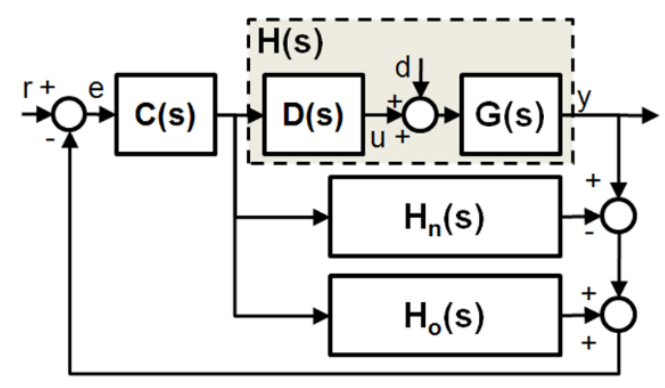

Figure 2. SP with decoupling scheme.

In some methods, the output prediction property of the SP scheme does not hold (Chen, He, \& Qi, 2011); in other cases, the time delay is not completely removed from the characteristic equation (Jerome \& Ray, 1986). The control system design for first order plus time delay systems was addressed in (Chen et al., 2011; Rao \& Chindambaram, 2006). In (Sánchez-Peña, Bolea, \& Puig, 2009), a robust control strategy is presented; however, it is limited to processes with time delays that can be factorized into input and output delays, and therefore, it cannot be used in presence of internal coupling delays. The main drawback of these methods is the use of the full model of the process to predict the non-delayed output. Therefore, they cannot be used to control unstable plants because the disturbance rejection response is governed by the open-loop dynamics. In addition, for stable processes, it is not possible to speed-up the disturbance rejection response to be faster than the open-loop dynamics. In (García \& Albertos, 2010), a control design procedure to deal with a particular case of unstable MIMO processes was presented. Recently, (Flesch, Torrico, Normey-Rico, \& Cavalcante, 2011) proposed a unified dead-time compensator for MIMO systems with multiple time delays. It is a generalization of the SISO filtered SP controller and it can be used to control stable or unstable dead-time processes and improve the disturbance rejection response. Later, in (Santos, Flesch, \& Normey-Rico, 2014), the ideas of the MIMO-FSP were 
extended to different delay-free models to understand the effect of the fast model in the closed-loop scheme.

From the SP scheme in Figure 1, the matrix expressions of the closed-loop transfer matrix T(s) from the references $r$ to the outputs $y$, and the transfer matrix $\mathrm{Q}(\mathrm{s})$ from the load disturbances $d$ to the outputs $y$ can be obtained as follows:

$$
\begin{aligned}
& T(s)=G(s) \cdot C(s) \cdot\left[I+\left(G(s)-G_{n}(s)+G_{o}(s)\right) \cdot C(s)\right]^{-1} \\
& Q(s)=G(s)-G(s) \cdot\left[I+C(s)\left(G(s)-G_{n}(s)+G_{o}(s)\right)\right]^{-1} \cdot C(s) \cdot G(s)
\end{aligned}
$$

where $\mathrm{G}(\mathrm{s}), \mathrm{G}_{\mathrm{n}}(\mathrm{s}), \mathrm{G}_{\mathrm{o}}(\mathrm{s})$ and $\mathrm{C}(\mathrm{s})$ are $n \times n$ transfer matrixes. When the nominal model of the process is perfect, i.e. $G_{n}(s)=G(s)$, the previous closed-loop transfer matrixes are simplified to (3) and (4).

$$
\begin{aligned}
& T(s)=G(s) \cdot C(s) \cdot\left[I+G_{o}(s) \cdot C(s)\right]^{-1} \\
& Q(s)=G(s)-G(s) \cdot\left[I+C(s) \cdot G_{o}(s)\right]^{-1} \cdot C(s) \cdot G(s)
\end{aligned}
$$

Then, the main controller C(s) can be calculated in order to obtain the desired performance in the closed-loop transfer matrix T(s) in (3). Its characteristic equation would be the determinant of $\left[I+G_{0}(s) \cdot C(s)\right]$, which only includes the fast model of the plant $G_{0}(s)$. In order to simplify this design, several methodologies design C(s) to obtain a diagonal matrix $\mathrm{G}_{0}(\mathrm{~s}) \cdot \mathrm{C}(\mathrm{s})$. Most of them use a transfer matrix $\mathrm{C}(\mathrm{s})$ in which the process inputs $u$ are derived by a time-weighted combination of the error signals $e$. If decoupling is required in T(s), the main problem of such methods is the increase of the design complication when the size of the system is large, because the calculations become more complex and important approximations are usually required. 
This work proposes a new tuning methodology of the main controller of a multivariable SP scheme for directly decoupling and stabilizing square multivariable processes with multiple time delays. It is based on the structure of centralized inverted decoupling (Garrido, Vázquez, \& Morilla, 2013) that allows obtaining very simple expressions for controller elements independently of the system size. However, as disadvantage, it cannot be applied to processes with multivariable zeros in the right half plane (RHP) since it results unstable. An initial version of this methodology was introduced only for stable $2 \times 2$ processes in (Garrido, Vázquez, \& Morilla, 2014c). In this work, further research was performed extending the method for $n \times n$ processes. The development is similar to that performed in (Garrido et al., 2014a), where the IMC approach and the inverted decoupling are applied to sable processes. This work gives some results that were not analyzed in previous work since it considers stable and unstable plants with multiple delays and studies the tuning of the primary controller of the proposed structure with has an important practical appeal. The paper is structured as follows. In Section 2, the proposed method is completely developed for $n \times n$ processes. Several aspects as realizability are discussed. The expressions for the particular case of stable processes in which all elements are first order plus time delay (FOPTD) systems are detailed. In order to improve disturbance rejection and apply the SP structure in unstable plants, a diagonal filter in the feedback loop is proposed. Section 3 illustrates the methodology with several simulation examples. Finally, conclusions are summarized in Section 4.

\section{Smith predictor with inverted decoupling}

\subsection{General expressions for $n \times n$ processes}

Assuming a square process $\mathrm{G}(\mathrm{s})$ with $n$ inputs and $n$ outputs and multiple time delays, the first step in order to apply the SP scheme in Figure 1 is defining the fast model of the plant $G_{0}(s)$. In this work, this fast model is proposed as the output fast model of $G_{n}(s)$ according to (5), 
where $\Theta(s)$ is a diagonal matrix that contains the minimal common time delays by outputs, that is, the minimal common time delays by row [6]. Then, $G_{0}(s)$ is calculated from (6). Notice that $\mathrm{G}_{0}(\mathrm{~s})$ may still contain multiple time delays; however, at least, one element by each row of $G_{0}(s)$ does not have time delay. Therefore, $G_{0}(s)$ is equal to $G(s)$ after extracting the minimal common time delays of each row.

$$
\begin{aligned}
& G_{n}(s)=\Theta(s) \cdot G_{o}(s) \\
& G_{o}(s)=\Theta^{-1}(s) \cdot G_{n}(s)
\end{aligned}
$$

As $\Theta(s)$ is a diagonal matrix, if $C(s)$ is designed to decouple $G_{0}(s)$, then, it also decouples $G_{n}(s)$, and diagonal matrixes of open-loop processes $L_{n}(s)$ and $L_{o}(s)$ are obtained, respectively. $L_{n}(s)$ is equal to $\Theta(s) \cdot L_{o}(s)$. $L_{n}(s)$ corresponds to the nominal open-loop process (with the time delays in $\Theta(s)$ ), and $\mathrm{L}_{\mathrm{o}}(\mathrm{s})$ corresponds to the nominal delay free open-loop process. Therefore, equations (3) and (4) would be reduced to (7) and (8) for the nominal case $\left(G_{n}(s)=G(s)\right)$.

$$
\begin{aligned}
& T(s)=\Theta(s) \cdot L_{o}(s) \cdot\left[I+L_{o}(s)\right]^{-1}=\Theta(s) \cdot T_{o}(s) \\
& Q(s)=G(s)-G(s) \cdot\left[I+L_{o}(s)\right]^{-1} \cdot C(s) \cdot G(s)
\end{aligned}
$$

According to (7), the closed-loop transfer matrix T(s) would be diagonal, which implies a decoupled response from the references to the outputs. Each closed-loop transfer function $t_{i}(s)$ would be given by (9). Consequently, it is possible to achieve the desired closed-loop performance defining the proper open-loop process $\mathrm{lo}_{\mathrm{i}}(\mathrm{s})$; and thanks to the SP structure, this transfer function $\mathrm{lo}_{\mathrm{i}}(\mathrm{s})$ is free of time delay, as it will be explained with details in section 2.2. Thus, the specification of performance requirements can be carried out easily. 
However, it is important to note that the closed-loop response is delayed by the corresponding time delay $\theta_{\mathrm{i}}$ of $\Theta(\mathrm{s})$.

$$
t_{i}(s)=t o_{i}(s) \cdot \mathrm{e}^{-s \cdot \theta i}=\frac{l_{i}(s)}{1+l_{i}(s)} \cdot \mathrm{e}^{-s \cdot \theta i}
$$

The proposed methodology uses a centralized inverted decoupling control to design the control matrix C(s) obtaining a decoupled response in $\mathrm{T}(\mathrm{s})$, which is achieved if the fastopen-loop transfer matrix $\mathrm{L}_{0}(\mathrm{~s})=\mathrm{G}_{0}(\mathrm{~s}) \cdot \mathrm{C}(\mathrm{s})$ is diagonal. Therefore, after specifying the desired delay-free elements of this diagonal fast open-loop transfer matrix $L_{0}(s)$ from the closed-loop specifications, the controller matrix C(s) must fulfils

$$
C(s)=G_{o}^{-1}(s) \cdot L_{o}(s)
$$

However, as it is shown in Figure 3, C(s) is split into two blocks: a matrix Cd(s) in the direct path (between the error signals $e$ and the control signals $u$ ) and a matrix $\operatorname{Co}(\mathrm{s})$ in a feedback loop (in the opposite direction). According to the inverted decoupling structure, Cd(s) must have only $n$ elements different from zero which connect the error signals $e$ with the control signals $u$. In order to decouple the system, Co(s) feeds back the control signals $u$ toward the controller inputs. Co(s) must have only $n$ zero elements, which correspond with the transpose non-zero elements of $\mathrm{Cd}(\mathrm{s})$. For example, in a $4 \times 4$ process, if element $\mathrm{Cd}(2,4)$ is selected as a direct connection between $u_{2}$ and $e_{4}$, there will not be feedback from $u_{2}$ to $e_{4}$ and consequently, $\operatorname{Co}(4,2)$ must be zero. 


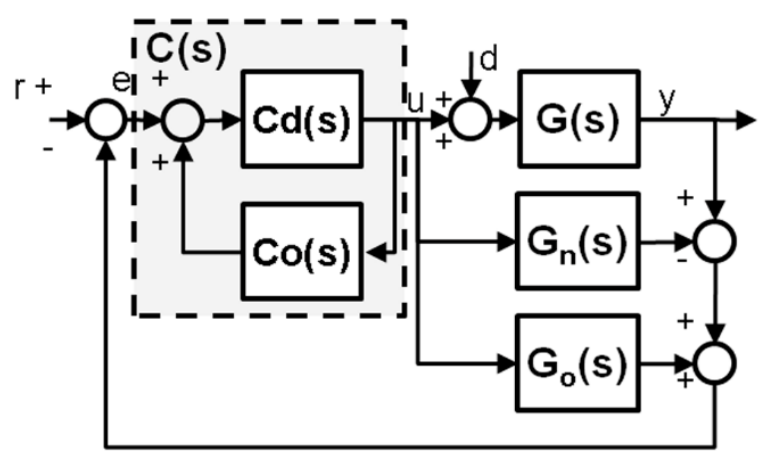

Figure 3. SP scheme with inverted decoupling.

From the representation in Figure 3, the expression of the whole controller matrix C(s) is calculated as follows:

$$
C(s)=C d(s) \cdot(I-C o(s) \cdot C d(s))^{-1}
$$

Combining the inverse of (10) with the inverse of (11), the simple expression in (12) is achieved, and it can be used to calculate $\mathrm{Cd}(\mathrm{s})$ and $\mathrm{Co}(\mathrm{s})$.

$$
C d^{-1}(s)-C o(s)=L_{o}^{-1}(s) \cdot G_{o}(s)
$$

Assuming that the desired closed-loop response is a decoupled response from the references to the outputs, the matrix $\mathrm{T}(\mathrm{s})$ must be diagonal and consequently, matrix $\mathrm{L}_{\mathrm{o}}(\mathrm{s})$ as well. Then, the main advantage of (12) is its simplicity, regardless process size, because the resulting subtraction of $\mathrm{Cd}^{-1}(\mathrm{~s})$ and $\mathrm{Co}(\mathrm{s})$ is a transfer matrix with only one element to be calculated for each position.

Note that $\mathrm{Cd}(\mathrm{s})$ has to be non-singular since it is inverted, and therefore, when its nonzero elements are chosen, only one element in each row and column can be selected. As a result, for $n \times n$ systems there are $n$ ! possible configurations of Cd(s). To name them, the authors propose a notation in which the indicated number corresponds to the column with the chosen element (Garrido, Vázquez, \& Morilla, 2011). For instance, in a 2×2 system there are two configurations: $1-2$ when elements $C d(1,1)$ and $C d(2,2)$ are selected to be non-zero; 2-1 
when elements $\mathrm{Cd}(1,2)$ and $\mathrm{Cd}(2,1)$ are chosen. The expression of the controller elements for each configuration is different, which is interesting because some choices can result in nonrealizable elements. Therefore, the configuration can be selected depending on the realizability, which will be discussed later.

The general expressions for $2 \times 2$ processes can be derived from (12) easily. For instance, assuming configuration 1-2, the following matrix equation is achieved:

$$
\begin{gathered}
\left(\begin{array}{cc}
c d_{11}(s) & 0 \\
0 & c d_{22}(s)
\end{array}\right)^{-1}-\left(\begin{array}{cc}
0 & c o_{12}(s) \\
c o_{21}(s) & 0
\end{array}\right)= \\
=\left(\begin{array}{cc}
\frac{1}{c d_{11}(s)} & -c o_{12}(s) \\
-c O_{21}(s) & \frac{1}{c d_{22}(s)}
\end{array}\right)=\left(\begin{array}{ll}
\frac{g o_{11}(s)}{l o_{1}(s)} & \frac{g o_{12}(s)}{l o_{1}(s)} \\
\frac{g o_{21}(s)}{l o_{2}(s)} & \frac{g o_{22}(s)}{l o_{2}(s)}
\end{array}\right)
\end{gathered}
$$

Then, the general expressions for the non-zero controller elements selecting configuration 1-2 are given by (14).

$$
\begin{aligned}
& c d_{11}(s)=\frac{l o_{1}(s)}{g o_{11}(s)} \quad c o_{12}(s)=\frac{-g o_{12}(s)}{l o_{1}(s)} \\
& c O_{21}(s)=\frac{-g o_{21}(s)}{l o_{2}(s)} \quad c d_{22}(s)=\frac{l o_{2}(s)}{g o_{22}(s)}
\end{aligned}
$$

In the same way, assuming configuration 2-1, the matrix equation in (10) is achieved and from it, the general expressions for the non-zero controller elements are given by (16).

$$
\begin{aligned}
& \left(\begin{array}{cc}
-c o_{11}(s) & \frac{1}{c d_{21}(s)} \\
\frac{1}{c d_{12}(s)} & -c o_{22}(s)
\end{array}\right)=\left(\begin{array}{ll}
\frac{g o_{11}(s)}{l o_{1}(s)} & \frac{g o_{12}(s)}{l o_{1}(s)} \\
\frac{g o_{21}(s)}{l o_{2}(s)} & \frac{g o_{22}(s)}{l o_{2}(s)}
\end{array}\right) \\
& c O_{11}(s)=\frac{-g o_{11}(s)}{l o_{1}(s)} \quad c d_{12}(s)=\frac{l o_{2}(s)}{g O_{21}(s)} \\
& c d_{21}(s)=\frac{l o_{1}(s)}{g o_{12}(s)} \quad c o_{22}(s)=\frac{-g o_{22}(s)}{l o_{2}(s)}
\end{aligned}
$$


In case of $3 \times 3$ processes, the procedure is the same: obtaining the expressions from (12) after choosing the configuration. In this case, there are six possible configurations according to the three elements selected in $\mathrm{Cd}(\mathrm{s})$ to be non-zero. For instance, choosing configuration 1-2-3 (diagonal elements of $\mathrm{Cd}(\mathrm{s})$ to be non-zero) in (12), the equation (17) is obtained. From this, the general expressions for the non-zero elements of $\mathrm{Cd}(\mathrm{s})$ and $\mathrm{Co}(\mathrm{s})$ in (18) are easily derived.

$$
\begin{aligned}
& \left(\begin{array}{ccc}
\frac{1}{c d_{11}(s)} & -c o_{12}(s) & -c O_{13}(s) \\
-c O_{21}(s) & \frac{1}{c d_{22}(s)} & -c O_{23}(s) \\
-c O_{31}(s) & -c O_{32}(s) & \frac{1}{c d_{33}(s)}
\end{array}\right)=\left(\begin{array}{lll}
\frac{g o_{11}(s)}{l o_{1}(s)} & \frac{g o_{12}(s)}{l o_{1}(s)} & \frac{g o_{13}(s)}{l o_{1}(s)} \\
\frac{g o_{21}(s)}{l o_{2}(s)} & \frac{g o_{22}(s)}{l o_{2}(s)} & \frac{g o_{23}(s)}{l o_{2}(s)} \\
\frac{g o_{31}(s)}{l o_{3}(s)} & \frac{g o_{32}(s)}{l o_{3}(s)} & \frac{g o_{33}(s)}{l o_{3}(s)}
\end{array}\right) \\
& c d_{11}(s)=\frac{l o_{1}(s)}{g o_{11}(s)} \quad c o_{12}(s)=\frac{-g o_{12}(s)}{l o_{1}(s)} \quad c o_{13}(s)=\frac{-g o_{13}(s)}{l o_{1}(s)} \\
& c O_{21}(s)=\frac{-g O_{21}(s)}{l O_{2}(s)} \quad c d_{22}(s)=\frac{l o_{2}(s)}{g O_{22}(s)} \quad c O_{23}(s)=\frac{-g O_{23}(s)}{l o_{2}(s)} \\
& c O_{31}(s)=\frac{-g O_{31}(s)}{l o_{3}(s)} \quad C O_{32}(s)=\frac{-g O_{32}(s)}{l o_{3}(s)} \quad c d_{33}(s)=\frac{l o_{3}(s)}{g O_{33}(s)}
\end{aligned}
$$

For higher dimensional systems, the procedure is similar. Therefore, from (12) it is possible to achieve the general expressions of the SP with inverted decoupling for $n \times n$ processes. If the configuration $p_{1}-p_{2}-\ldots-p_{i}-\ldots-p_{n-1}-p_{n}$ is chosen, the non-zero elements of the Cd(s) and Co(s) matrices are provided in (19) and (20), respectively. The transfer functions of the desired fast open-loop processes $\mathrm{lo}_{i}(\mathrm{~s})$ can be specified in any way that assures the realizability of these controller elements, as discussed in section 2.2

$$
\begin{aligned}
& c d_{i j}(s)=\frac{l o_{j}(s)}{g o_{j i}(s)} \quad \forall i ; j=p_{i} \\
& C O_{i j}(s)=\frac{-g o_{i j}(s)}{l o_{i}(s)} \quad \forall i, j ; / i \neq p_{j}
\end{aligned}
$$


From these general expressions, it can be concluded that the complexity of the controller elements in $\mathrm{Cd}(\mathrm{s})$ and $\mathrm{Co}(\mathrm{s})$ is always the same, independent of the system size. With conventional schemes in $\mathrm{C}(\mathrm{s})$, these elements become more complex as the size of the process increases. However, it is necessary to indicate that the proposed methodology has the same disadvantage as inverted decoupling: it cannot be applied to processes with multivariable RHP zeros, that is, RHP zeros in its determinant (Garrido et al., 2011, 2013). If a RHP zero is specified in some $l o_{i}(\mathrm{~s})$ transfer function, it will appear as unstable pole in some $\mathrm{CO}_{i j}(\mathrm{~s})$ elements. The case of RHP zeros associated to a single output is an exception. In this case, although the RHP zero is specified in the corresponding fast open-loop transfer function $l o_{i}(\mathrm{~s})$, it will be canceled in the controller elements (Garrido et al., 2011).

\subsection{Realizability}

The realizability requirement for $\mathrm{Cd}(\mathrm{s})$ and $\mathrm{Co}(\mathrm{s})$ is that all of their elements must be stable, causal and proper. For processes with time delays, RHP zeros or RHP poles, direct calculations can lead to elements with prediction or unstable poles. In the proposed methodology, there are two issues regarding controller realizability that have to be analyzed: firstly, it is necessary to check if it is possible to achieve realizability using the selected configuration; and secondly, after confirming the previous condition, it is essential to determine how to specify the desired fast open-loop transfer functions $l_{i}(\mathrm{~s})$. Next, the conditions that a configuration needs to fulfill to be realizable are provided. In addition, the constraints on the open-loop processes to achieve such realizability are indicated as well.

In the controller expressions (19) and (20), each desired fast open-loop transfer function $l o_{i}(\mathrm{~s})$ appears associated to the fast process transfer functions $g o_{i j}(\mathrm{~s})$ of the same row $i$. Thus, there are four aspects to take into consideration and to be inspected for each row of the fast model of the plant $G_{0}(s)$ : 
- Non-causal time delays must be avoided in controller elements. As $G_{0}(s)$ is the output fast model of the plant, at least, one element by row of $\mathrm{G}_{0}(\mathrm{~s})$ does not have any time delay. If $g o_{i k}(\mathrm{~s})$ is the transfer function of the row $i$ without delay, the element $c d_{k i}(\mathrm{~s})$ of $\mathrm{Cd}(\mathrm{s})$ should be chosen to be in the direct path between the error signals and the control signals (it should be non-zero). This transfer function $g o_{i k}(\mathrm{~s})$ will appear in the denominator of the corresponding controller element $c d_{k i}(\mathrm{~s})$ according to (19). The numerator of this controller element is given by the desired fast open-loop transfer function $l o_{i}(\mathrm{~s})$ which must have no delay. In contrast, according to (20), $l o_{i}(\mathrm{~s})$ appears in the denominator of the corresponding controller elements $c o_{i j}(\mathrm{~s})$. Although, the other $g o_{i j}(\mathrm{~s})$ elements different from $g o_{i k}(\mathrm{~s})$ in the row $i$ can have time delay, this delay will appear in the numerator avoiding non-causal time delays in $\mathrm{co}_{i j}(\mathrm{~s})$. If the only elements by row of $\mathrm{G}_{\mathrm{o}}(\mathrm{s})$ without time delay are necessarily in the same column (that is, the minimal output time delays are in the same column), then, the nominal process $G_{n}(s)$ should be modified adding some extra time delay, as discussed later.

- Controller elements must be proper, that is, their relative degree must be greater or equal than zero. If $g o_{i k}(\mathrm{~s})$ is the transfer function of the row $i$ with the smallest relative degree $r_{i k}$, the element $c d_{k i}(\mathrm{~s})$ should be different from zero to avoid improper controller elements. In addition, the relative degree $r_{i}$ of the fast open-loop process $l o_{i}(\mathrm{~s})$ must fulfill:

$$
r_{i k} \leq r_{i} \leq \min _{j \neq k}\left(r_{i j}\right)
$$

- Controller elements must be stable. When an individual RHP zero appears in a transfer function $g o_{i m}(\mathrm{~s})$, the element $c d_{m i}(\mathrm{~s})$ of $\mathrm{Cd}(\mathrm{s})$ should not be selected in the direct path, in order to avoid this zero becomes an unstable pole in this element. When the RHP zero appears in all elements of the same row, it is necessary to check its multiplicity in 
each element of the row. Once more, if $g o_{i k}(\mathrm{~s})$ is the process transfer function of the row $i$ with the smallest RHP zero multiplicity $\eta_{i k}$, the element $c d_{k i}(\mathrm{~s})$ should be chosen to be in the direct path (it should be non-zero). This RHP zero must appear in the open-loop process $l_{i}(\mathrm{~s})$ with a multiplicity $\eta_{i}$ that fulfills (22). This condition must be fulfilled for each different RHP zero $z_{x}$ of the row $i$.

$$
\eta_{i k} \leq \eta_{i} \leq \min _{j \neq k}\left(\eta_{i j}\right)
$$

- In line with the previous case, when an individual RHP pole appears in a transfer function $g o_{i m}(\mathrm{~s})$, the element $c d_{m i}(\mathrm{~s})$ of $\mathrm{Cd}(\mathrm{s})$ should be selected in the direct path and this unstable pole must be specified in the corresponding transfer function $l o_{i}(\mathrm{~s})$, in order to avoid an unstable controller element. When the pole appears in several elements of the same row, it is necessary to check its multiplicity in each element of the row. In this case, if $g o_{i k}(\mathrm{~s})$ is the process transfer function of the row $i$ with the largest RHP pole multiplicity $\psi_{i k}$, the element $c d_{k i}(\mathrm{~s})$ should be chosen to be in the direct path (it should be non-zero). This RHP pole must appear in the open-loop process $l_{i}(\mathrm{~s})$ with a multiplicity $\psi_{i}$ that fulfills (23). This condition must be fulfilled for each different RHP pole $p_{x}$ of the row $i$.

$$
\psi_{i k} \geq \psi_{i} \geq \max _{j \neq k}\left(\psi_{i j}\right)
$$

It is important to realize that the SP scheme of Figure 3 cannot be directly apply in unstable plants. The blocks $\mathrm{G}_{\mathrm{n}}(\mathrm{s})$ and $\mathrm{G}_{\mathrm{o}}(\mathrm{s})$ would contain unstable elements and consequently, they will be internally unstable. As it is discussed later in section 2.5, another implementation scheme is proposed with an additional filter that allows obtaining a stable output prediction for reject step disturbance and achieving internally stability. 
When two or more elements of Cd(s) have to be selected necessarily in the same column to satisfy the previous conditions in all rows, there is no realizable configuration. Then, it is necessary to insert an additional block N(s) between the system G(s) and the inverted decoupling controller in order to modify the process and to force the non-realizable elements into realizability. Then, the proposed method of SP with inverted decoupling would be applied to the new process $\mathrm{G}^{\mathrm{N}}(\mathrm{s})=\mathrm{G}(\mathrm{s}) \cdot \mathrm{N}(\mathrm{s})$.

$\mathrm{N}(\mathrm{s})$ is a diagonal transfer matrix with the necessary extra dynamics. If there are no realizability problems in the row $i$, the $n_{i i}(\mathrm{~s})$ element is equal to the unity. Otherwise, the required extra dynamics (time delay, pole or RHP zero) is added with the proper multiplicity to fulfill the corresponding realizability condition. In general, $n_{i i}(\mathrm{~s})$ is defined according to (24). Generally, it is preferable to add the minimum extra dynamics. Therefore, after checking the required additional dynamics of each configuration, it is selected that one with fewer RHP zeros, poles or time delays in N(s). More detailed information about this issue is provided in (Garrido et al., 2011). However, note that RHP poles have not been included in this expression in order to avoid an unstable transfer function. Therefore, if condition (23) cannot be initially fulfilled in all rows, there are no realizable configurations.

$$
n_{i i}(\mathrm{~s})=e^{-\theta_{i i} s} \cdot \frac{1}{(\tau s+1)^{r_{i i}}} \cdot \prod_{x=1}^{N z}\left(\frac{-s+z_{x}}{s+z_{x}}\right)^{n x_{i i}}
$$

\subsection{How to specify the desired delay free open-loop processes $1 \mathrm{l}_{\mathrm{i}}(\mathrm{s})$}

For a given configuration, the four previous conditions must be fulfilled for realizability when $l_{i}(\mathrm{~s})$ is specified. Nevertheless, for best performance of the control system, it is undesirable to include any RHP zero or RHP pole in $l o_{i}(\mathrm{~s})$ more than necessary. Therefore, $l o_{i}(\mathrm{~s})$ is defined with the minimum RHP pole multiplicity and minimum RHP zero multiplicity which 
fulfill the realizability conditions (22) and (23), that is, the minimum values by row $i$. In addition, since the closed-loop response must be stable and without steady-state errors due to set point or load changes, $l o_{i}(\mathrm{~s})$ must contain an integrator. The following form of $l o_{i}(\mathrm{~s})$ is suggested:

$$
l_{i}(\mathrm{~s})=\frac{1}{\lambda_{i} s} \prod_{x=1}^{N z}\left(\frac{-s+z_{x}}{s+z_{x}}\right)^{n x_{i}} \cdot \prod_{x=1}^{N p}\left(\frac{s+p_{x}}{s-p_{x}}\right)^{\mu x_{i}} \cdot \frac{1}{\left(\tau_{i} s+1\right)^{r_{i}-1}}
$$

where the time constant $\lambda_{i}$ determines the bandwidth of the closed-loop $i$ and acts as a tuning parameter for performance and robustness, $\mathrm{Nz}$ is the total number of individual RHP zeros of the row $i$, and $\eta x_{i}$ is the proper multiplicity of the zero $z_{X}$ according to condition (22). $N p$ is the total number of individual RHP poles of the row $i$, and $\psi x_{i}$ is the proper multiplicity of the pole $p_{x}$ according to condition (23). The multiplicity $r_{i}$ of the associated pole is specified to provide the necessary high frequency roll-off rate being limited by the realizability condition (21). When $l o r(s)$ must have zero relative degree according to (21), a zero can be included and used as an extra tuning parameter.

Next, three particular cases of $l_{i}(\mathrm{~s})$ transfer functions are shown. They are collected in Table 1 . The first case arises when $l o_{i}(\mathrm{~s})$ can be defined as an integrator with a constant according to the previous realizability conditions. The closed-loop transfer function has the typical shape of a first order system with time constant $\lambda_{i}$ plus the corresponding output time delay of $G(s)$.

Table 1. Three common cases to define the delay free open-loop transfer functions.

\begin{tabular}{ccc}
\hline Case & $l_{i}(\mathrm{~s})$ & $t_{i}(\mathrm{~s})$ \\
\hline 1 & $\frac{1}{\lambda_{i} s}$ & $\frac{1}{\lambda_{i} s+1} \cdot e^{-s \theta i}$ \\
2 & $\frac{1}{\lambda_{i} \cdot s \cdot\left(\tau_{i} \cdot s+1\right)}$ & $\frac{1}{\lambda_{i} \tau_{i} s^{2}+\lambda_{i} s+1} \cdot e^{-s \theta i}$ \\
3 & $\frac{\left(s+p_{i}\right)}{\lambda_{i} \cdot s \cdot\left(s-p_{i}\right)}$ & $\frac{\left(\mathrm{s}+p_{i}\right) / \lambda_{i}}{s^{2}+\left(1 / \lambda_{i}-p_{i}\right) s+p_{i} / \lambda_{i}} \cdot e^{-s \theta i}$ \\
\hline
\end{tabular}


Second, when a relative degree equal to two must be specified in $l_{i}(\mathrm{~s})$ without any RHP zero, it is necessary to include a pole in $s=-1 / \tau_{\mathrm{i}}$ according to (25). Then, the closed-loop transfer function is obtained as a second order system plus time delay. The poles of this $t_{i}(\mathrm{~s})$ are characterized by the undamped natural frequency $\omega_{\mathrm{n}}$ and the damping factor $\xi$ given by (26).

$$
\omega_{n}=1 / \sqrt{\tau_{i} \lambda_{i}} \quad \xi=0.5 \sqrt{\lambda_{i} / \tau_{i}}
$$

The third row of Table 1 shows the case in which a RHP pole $s=p_{i}$ must be specified in $l_{i}(\mathrm{~s})$ and relative degree one is required. The corresponding closed-loop transfer function is a second order system plus time delay and zero at $\mathrm{s}=-p_{i}$. The poles of this $t_{i}(\mathrm{~s})$ are characterized by the undamped natural frequency $\omega_{\mathrm{n}}$ and the damping factor $\xi$ given by (27). In order to obtain a stable closed-loop transfer function, the condition $\lambda_{i}<1 / p_{i}$ must be fulfilled. From the second expression in (27), the $\lambda_{i}$ value for a desired damping factor is given by (28). In order to avoid the effect of the cero at $s=-1 / p_{i}$ in the reference tracking response $t_{i}(\mathrm{~s})$, a reference filter can be used containing this zero as a pole with a unitary stationary gain.

$$
\begin{aligned}
& \omega_{n}=\sqrt{p_{i} / \lambda_{i}} \quad \xi=\frac{0.5 \cdot\left(1-\lambda_{i} \cdot p_{i}\right)}{\sqrt{\lambda_{i} \cdot p_{i}}} \\
& \lambda_{i}=\left(\frac{-\xi+\sqrt{\xi^{2}+1}}{\sqrt{p_{i}}}\right)^{2}
\end{aligned}
$$

\subsection{Application to stable MIMO systems with FOPTD elements}

Since almost all industry processes are open-loop stable and exhibit non oscillatory response for step inputs, higher order transfer functions can be simplified to a first order plus time 
delay (FOPDT) model before the control design. Thus, the process elements $g_{i j}(\mathrm{~s})$ are given by a stable transfer function as follows:

$$
g_{i j}(s)=\frac{K_{i j}}{T_{i j} s+1} e^{-s \theta i j}
$$

Assuming a realizable configuration $p_{1}-p_{2}-\ldots-p_{i}-\ldots-p_{n-1}-p_{n}$ in a $n \times n$ process, realizability conditions point out that each open-loop transfer function $l_{i}(\mathrm{~s})$ has a relative degree equal to the unity, no RHP zeros and no RHP poles. Consequently, $l_{i}(\mathrm{~s})$ can be specified as the case 1 of Table 1.

Assuming $G(s)=G_{n}(s)$, the controller elements of $C d(s)$ and $C o(s)$ are obtained as shown in (30) and (31) according to (19) and (20), respectively. The elements of Cd(s) result PI controllers, and those of Co(s) are filtered-derivative compensators plus time delay.

$$
\begin{aligned}
& c d_{i j}(s)=\frac{1}{K_{j i}} \cdot \frac{\left(T_{j i} s+1\right)}{\lambda_{j} s}=\frac{K_{P i j} s+K_{I i j}}{s} \quad \forall i ; \quad j=p_{i} \\
& C O_{i j}(s)=-K_{i j} \cdot \frac{\lambda_{i} s}{\left(T_{i j} s+1\right)} \cdot e^{-s(\theta i j-\theta i)}=\frac{K_{D i j} s}{N_{i j} s+1} e^{-s \theta\left(\left(c_{i j}\right)\right.} \quad \forall i, j ; / i \neq p_{j}
\end{aligned}
$$

From (30) and (31), it can be found that the controller parameters are given by

$$
\begin{aligned}
& K_{P i j}=T_{j i} /\left(\lambda_{j} K_{j i}\right) \quad ; \quad K_{I i j}=1 /\left(\lambda_{j} K_{j i}\right) \quad \forall i ; \quad j=p_{i} \\
& K_{D i j}=-\lambda_{i} K_{i j} \quad ; \quad N_{i j}=T_{i j} \quad ; \theta\left(c o_{i j}\right)=\theta_{i j}-\theta_{i} \quad \forall i, j ; / i \neq p_{j}
\end{aligned}
$$

When it is possible to approximate the transfer functions of the process by stable FOPTD systems, authors propose to do it and to use the simple equations (32) to calculate the controller parameters.

\subsection{Additional filter}

According to (2), the disturbance rejection performance is governed by the open-loop 
dynamics of the process $G(s)$. In order to improve the disturbance rejection response of the closed-loop system, a stable diagonal filter $\mathrm{F}(\mathrm{s})$ is proposed as shown in the scheme of Figure 4. A similar filter is proposed in the multivariable filtered Smith predictor in (Flesch et al., 2011). Then, the following closed-loop transfer matrixes T(s) and Q(s) are obtained (where Laplace variable $s$ has been omitted):

$$
\begin{aligned}
& T=G \cdot C \cdot\left[I+F\left(G-G_{n}+F^{-1} G_{o}\right) \cdot C\right]^{-1} \\
& Q=G-G \cdot\left[I+C \cdot F\left(G-G_{n}+F^{-1} G_{o}\right)\right]^{-1} \cdot C \cdot F \cdot G
\end{aligned}
$$

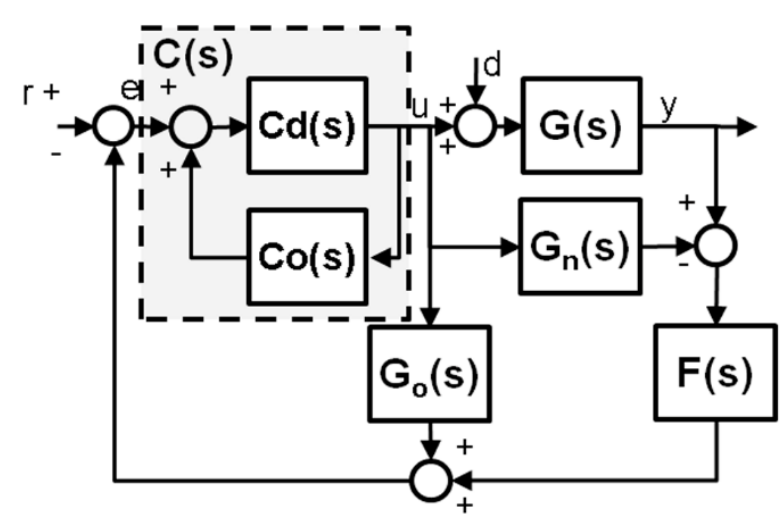

Figure 4. Smith predictor with inverted decoupling plus filter (for analysis).

For the nominal case $\left(G(s)=G_{n}(s)\right)$, the reference tracking response remains the same as the one obtained without the filter, independent of F(s). Nevertheless, the load disturbance response is modified by the filter as follows:

$$
Q=G-G \cdot\left[I+L_{o}\right]^{-1} C \cdot F \cdot G=(I-T \cdot F) \cdot G
$$

Since $\mathrm{T}(\mathrm{s})$ and $\mathrm{F}(\mathrm{s})$ are diagonal matrixes, matrix $[\mathrm{I}-\mathrm{T}(\mathrm{s}) \cdot \mathrm{F}(\mathrm{s})]$ is diagonal as well. In order to cancel the undesired poles of each row $i$ of a stable process $G(s)$, the filter element $f_{i}(\mathrm{~s})$ must be designed in such a way that these slow poles appear as zeros in $\left(1-t_{i}(\mathrm{~s}) \cdot f_{i}(\mathrm{~s})\right)$. This is satisfied if the following condition is fulfilled: 


$$
\left.\frac{d^{r}}{d s^{r}}\left(1-t_{i}(s) \cdot f_{i}(s)\right)\right|_{s=z_{k}}=0 \quad r=0,1, \ldots, m_{k}-1 ; k=1, \ldots, p
$$

where $z_{k}$ is an undesired pole, $m_{k}$ is its maximum multiplicity in the row $i$ of $G(s)$, and $p$ is the total number of undesired poles in the row $i$. In general, the filter element $f_{i}(\mathrm{~s})$ is defined as follows:

$$
f_{i}(s)=\frac{N_{\alpha}(s) \cdot\left(\lambda_{i} s+1\right)^{r}}{\left(\beta_{i} s+1\right)^{\eta}}
$$

where the term $\left(\lambda_{\mathrm{i}} \mathrm{s}+1\right)^{\mathrm{r}}$ cancels in $q_{i}(\mathrm{~s})$ the specified closed-loop pole or poles for reference tracking. The pole in $s=-1 / \beta_{i}$ is used to define the desired time constant of the disturbance rejection response and its degree must be chosen to obtain a proper filter element. Note also that, according to (35), the stationary gain of $f_{i}(\mathrm{~s})$ must be equal to the unity in order to obtain zero steady state error for step disturbance rejection. Then, $\mathrm{N}_{\alpha}(\mathrm{s})=\left[\alpha_{v} \mathrm{~s}+\ldots+\alpha_{1} \mathrm{~s}+1\right]$ is a polynomial of the proper degree $v$ and coefficients $\alpha_{k}$ which must be calculated in such a way that $\left[1-t_{i}(\mathrm{~s}) \cdot f_{i}(\mathrm{~s})\right]$ has the undesired poles of the original disturbance rejection response as zeros.

If $t_{i}(\mathrm{~s})$ is given by a stable FOPTD system with an only undesired pole at $s=-\mathrm{z}_{1}$ in $q_{i}$ (s), the filter is usually defined by (38) and $\alpha_{1}$ is calculated according to (39).

$$
\begin{aligned}
& f_{i}(s)=\frac{\left(\alpha_{1} s+1\right)\left(\lambda_{i} s+1\right)}{\left(\beta_{i} s+1\right)^{2}} \\
& \alpha_{1}=\left[1-\left(1-\beta_{i} \cdot z_{1}\right)^{2} \cdot e^{-\theta_{i} z_{1}}\right] / z_{1}
\end{aligned}
$$

As it is shown in (Flesch et al., 2011; Normey-Rico \& Camacho, 2009), the filter can also be used to improve the robustness of the system. However, it is shown that there is a trade-off between robustness and disturbance rejection performance. When $\beta_{i}$ decreases, the 
disturbance rejection becomes faster and the robustness deteriorates, and vice versa. This compromise must be studied for each case. In addition, the filter element $f_{i}(\mathrm{~s})$ can be specified with more poles than zeros to provide low pass behaviour. This reduces the high frequency noise and can increase the robustness.

Another important use of the diagonal filter $\mathrm{F}(\mathrm{s})$ is related to unstable processes (Flesch et al., 2011). Note that the SP structure in Figure 4 must be used just for analysis or stable processes. If it is used for implementation in unstable cases, the controller will be internally unstable since the blocks $G_{n}(s)$ or $G_{0}(s)$ will be unstable. In (Flesch et al., 2011), the scheme of Figure 5 is proposed for implementation in these cases, where block S(s) is stable and given by

$$
S(s)=(I-F(s) \cdot \Theta(s)) \cdot G_{o}(s)
$$

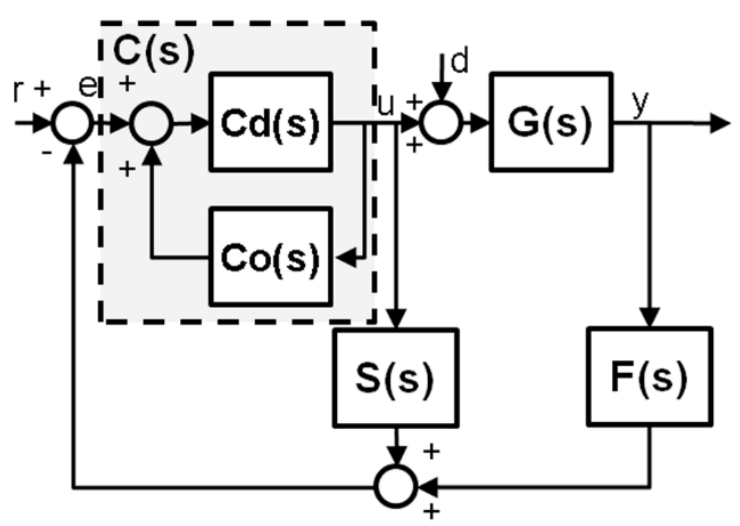

Figure 5. Smith predictor with inverted decoupling plus filter (for implementation).

The stable diagonal filter must be designed to eliminate any unstable pole of $G_{0}(s)$ from the elements of S(s). In this way, the filter design allows to obtain an open-loop-stable prediction of the output. Therefore, the unstable poles of row $i$ of $G_{0}(s)$ must appear as zeros in $\left(1-e^{-\theta \cdot s} \cdot f_{i}(\mathrm{~s})\right)$. This is satisfied if the following condition is fulfilled (Flesch et al., 2011):

$$
\left.\frac{d^{r}}{d s^{r}}\left(1-e^{-\theta_{i} s} \cdot f_{i}(s)\right)\right|_{s=p_{k}}=0 \quad r=0,1, \ldots, n_{k}-1 ; k=1, \ldots, p
$$


where $\mathrm{p}_{k}$ is an unstable pole, $n_{k}$ is its maximum multiplicity in the row $i$ of $\mathrm{G}_{0}(\mathrm{~s})$, and $p$ is the total number of undesired poles in row $i$. In general, this design is performed projecting the controller in the discrete domain, since the time delays have a polynomial representation in $\mathrm{z}$. In any case, the block $\mathrm{S}$ is easier to implement in the discrete domain.

The proposed SP scheme with inverted decoupling of Figure 5 can be transformed into a centralized inverted decoupling scheme, which can be interesting for an alternative implementation point of view. To do so, the block S(s) and Co(s) are combined into an only matrix $\operatorname{Ko}(\mathrm{s})=\mathrm{Co}(\mathrm{s})-\mathrm{S}(\mathrm{s})$ in the same position of $\mathrm{Co}(\mathrm{s})$. For a particular configuration $p_{1-} p_{2}$ $\ldots-p_{i}-\ldots-p_{n-1}-p_{n}$ and assuming $G_{n}(s)=G(s)$, the elements of Ko(s) would be given by (42) and (43). The elements in (42) correspond to the zero elements of Co(s). The implementation is also easier in the discrete domain.

$$
\begin{aligned}
& k o_{i j}(s)=-s_{i j}(s)=-g o_{i j}(s) \cdot\left(1-e^{-\theta_{i} s} \cdot f_{i}(s)\right) \quad \forall j ; \quad i=p_{j} \\
& k o_{i j}(s)=c o_{i j}(s)-s_{i j}(s)=\frac{-g o_{i j}(s)}{l o_{i}(s)} \cdot\left(1+l o_{i}(s)-e^{-\theta_{i} s} \cdot f_{i}(s) \cdot l_{i}(s)\right) \quad \forall i, j ; \quad / \quad i \neq p_{j}
\end{aligned}
$$

\section{Examples}

In this section, three simulation examples are presented to demonstrate the effectiveness of the proposed methodology. Two examples are stable systems, and the third one is a $2 \times 2$ unstable case.

\subsection{Example 1: Wardle and Wood distillation column}

The transfer function matrix of this $2 \times 2$ process is given by (44) in (Rao, Rao, \& Chidambaram, 2007). The time constants and delays are expressed in minutes. The process does not have multivariable RHP zeros. 


$$
G_{w W}(s)=\left(\begin{array}{cc}
\frac{0.126 \cdot e^{-6 s}}{60 s+1} & \frac{-0.101 \cdot e^{-12 s}}{(48 s+1)(45 s+1)} \\
\frac{0.094 \cdot e^{-8 s}}{38 s+1} & \frac{-0.12 \cdot e^{-8 s}}{35 s+1}
\end{array}\right)
$$

According to the conditions about time delays and relative degrees of section 2.2, configuration 1-2 must be chosen for realizability without adding extra dynamics. The common output delays are obtained as $\theta_{1}=6 \mathrm{~min}$ and $\theta_{2}=8 \mathrm{~min}$. Therefore, the fast model $G_{o}(\mathrm{~s})$ is given by:

$$
G_{0}(s)=\left(\begin{array}{cc}
\frac{0.126}{60 s+1} & \frac{-0.101 \cdot e^{-6 s}}{(48 s+1)(45 s+1)} \\
\frac{0.094}{38 s+1} & \frac{-0.12}{35 s+1}
\end{array}\right)
$$

The two desired open-loop process $l o_{i}(\mathrm{~s})$ can be specified as $1 /\left(\lambda_{i} s\right)$ according to case 1 of Table 1 , and consequently, the closed-loop transfer functions $t_{i}(\mathrm{~s})$ are given by FOPTD systems. After chosen the closed-loop time constants $\lambda_{1}=\lambda_{2}=15 \mathrm{~min}$, the controller elements are calculated by means of expressions (14). The resultant elements are:

$$
\begin{aligned}
& C d(s)=\left(\begin{array}{cc}
\frac{60 s+1}{1.89 s} & 0 \\
0 & \frac{-35 s-1}{1.8 s}
\end{array}\right) \\
& C o(s)=\left(\begin{array}{cc}
0 & \frac{1.515 s \cdot \mathrm{e}^{-6 s}}{(48 \mathrm{~s}+1)(45 s+1)} \\
\frac{-1.41 \mathrm{~s}}{38 s+1} & 0
\end{array}\right)
\end{aligned}
$$

The closed-loop system response of the proposed control using the scheme of Figure 3 (SPID) is shown in Figure 6. There is unit step changes at $t=0 \mathrm{~min}$ in the first set point, and at $t=500 \mathrm{~min}$, in the second one. There are also a -20 step change at $t=1000 \mathrm{~min}$ in both inputs as 
load disturbances. For comparison, the decentralized Smith Predictor (D-SP) control proposed in (Rao et al., 2007) is also shown.

The proposed controller achieves perfect decoupling. The proposed controller and the D-SP control obtains similar performance and IAE values in the first loop; however, the proposed control improves the response of the second loop with smaller settling time and IAE value (see Table 2), and better disturbance rejection.

The disturbance rejection in the first loop is a bit slow compared to that of the second loop. Therefore, a filter $f_{1}(\mathrm{~s})$ is designed in order to speed up this rejection. The slowest pole associated to the first output in $q_{11}(\mathrm{~s})$ and $q_{12}(\mathrm{~s})$ is $s=-1 / 60$. The filter is calculated according to (38) and (39) to cancel this pole. The time constant of the disturbance rejection response $\beta_{1}$ is fixed equal to $\lambda_{1}$. The diagonal filter $F(\mathrm{~s})$ is given by

$$
\mathrm{F}(\mathrm{s})=\left(\begin{array}{cc}
\frac{29.46 s+1}{15 s+1} & 0 \\
0 & 1
\end{array}\right)
$$

The closed-loop response of the proposed control with filter (SPID-F) is also shown in Figure 6 . The reference tracking response remains the same, and the disturbance rejection in the first loop is improved obtaining a smaller IAE value, similar to that of the second loop.
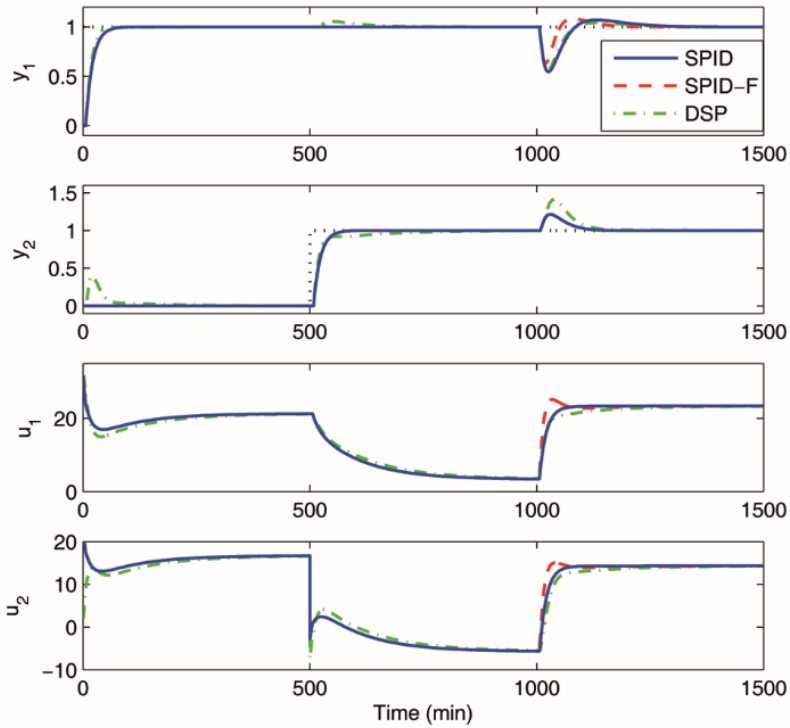
Figure 6. Outputs and control signals of the step response in example 1.

Table 2. Performance and robustness indices for each method in example 1.

\begin{tabular}{|c|c|c|c|c|c|c|}
\hline & \multicolumn{2}{|c|}{ SPID } & \multicolumn{2}{|c|}{ SPID-F } & \multicolumn{2}{|r|}{ D-SP } \\
\hline & loop & loop & loop & 1 loop & loop & 1 loop 2 \\
\hline IAE & 48.5 & 35.1 & 36.5 & 35.1 & 46.4 & 76.8 \\
\hline TV & 56.9 & 50.1 & 61.7 & 52.4 & 63.1 & 65.1 \\
\hline $\boldsymbol{\mu}_{\mathrm{RS}}$ & & .20 & & 0.26 & & 0.22 \\
\hline$\mu_{\mathrm{RP}}$ & & .83 & & 1.01 & & 1.03 \\
\hline
\end{tabular}

In order to evaluate the robustness of the controllers, a $\mu$-analysis is performed in presence of diagonal multiplicative input uncertainty. Multiplicative input uncertainty is represented as illustrated in Figure 7, where $\Delta_{\mathrm{I}}(\mathrm{s})$ is the disturbance and $\mathrm{W}_{\mathrm{I}}(\mathrm{s})$ and $\mathrm{W}_{\mathrm{P}}(\mathrm{s})$ are the diagonal weights for uncertainty and performance, respectively.

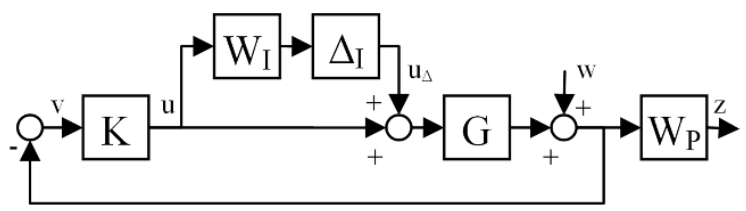

Figure 7. System with multiplicative input uncertainty and performance measured at the output.

To achieve robust stability the necessary and sufficient condition in a classical feedback system (Skogestad \& Postlethwaite, 2005) is

$$
\mu_{\mathrm{RS}}=\mu\left[-\mathrm{W}_{\mathrm{I}}(\mathrm{s}) \mathrm{T}_{\mathrm{I}}(\mathrm{s})\right]<1 \quad \forall \omega
$$

where $\mu$ is the structured singular value (SSV) and $T_{I}(s)=K(s) G(s)(I+K(s) G(s))^{-1}$ is the input complementary sensitivity function. To evaluate if the closed-loop system will respect the desired performance even in presence of diagonal multiplicative input uncertainty, the necessary and sufficient condition (Skogestad \& Postlethwaite, 2005) is 


$$
\mu_{\mathrm{RP}}=\mu\left[\begin{array}{cc}
-\mathrm{W}_{\mathrm{I}}(\mathrm{s}) \mathrm{T}_{\mathrm{I}}(\mathrm{s}) & -\mathrm{W}_{\mathrm{I}}(\mathrm{s}) \mathrm{K}(\mathrm{s}) \mathrm{S}(\mathrm{s}) \\
\mathrm{W}_{\mathrm{P}}(\mathrm{s}) \mathrm{S}(\mathrm{s}) \mathrm{G}(\mathrm{s}) & \mathrm{W}_{\mathrm{P}}(\mathrm{s}) \mathrm{S}(\mathrm{s})
\end{array}\right]<1 \quad \forall \omega
$$

where $S(s)=(I+G(s) K(s))^{-1}$ is the sensitivity function and $T(s)=G(s) K(s)(I+G(s) K(s))^{-1}$ is the complementary sensitivity function.

To carry out this analysis, it is necessary to calculate the equivalent conventional centralized feedback controller K(s) according to the structure of Figure 7, and to define the weights. In this example, the chosen weights are

$$
\begin{aligned}
& W_{I}(s)=W_{I}(s) \cdot I=\frac{(1.5 \cdot s+0.2)}{s+1} \cdot I \\
& W_{P}(s)=W_{P}(s) \cdot I=\frac{(s / 2+0.01)}{s} \cdot I
\end{aligned}
$$

The weight $w_{I}(\mathrm{~s})$ can be loosely interpreted as the process inputs increase by up to $150 \%$ uncertainty at high frequencies and by almost $20 \%$ uncertainty in the low frequency range. The performance weight $w_{P}(\mathrm{~s})$ specifies integral action, a maximum peak for the singular value of the sensitivity transfer matrix of $\mathrm{M}_{\mathrm{s}}=2$ and a bandwidth of about 0.01 $\mathrm{rad} / \mathrm{min}$.

The SSV for robust stability (RS) and robust performance (RP) for the different controllers are shown in Figure 8. The proposed SPID control obtains the best robust stability and robust performance. They fulfill conditions (49) and (50) for all frequencies, indicating that the system will remain stable in spite of an uncertainty of $20 \%$ on each process input. The peak values are shown in Table 2. For the proposed SPID-F control, the RS and RP will deteriorate at middle frequencies, where the peaks appear, because there is a trade-off between robustness and disturbance rejection, as it was mentioned at the end of section 2 (Flesch et al., 2011). These peak values are also collected in Table 2. 

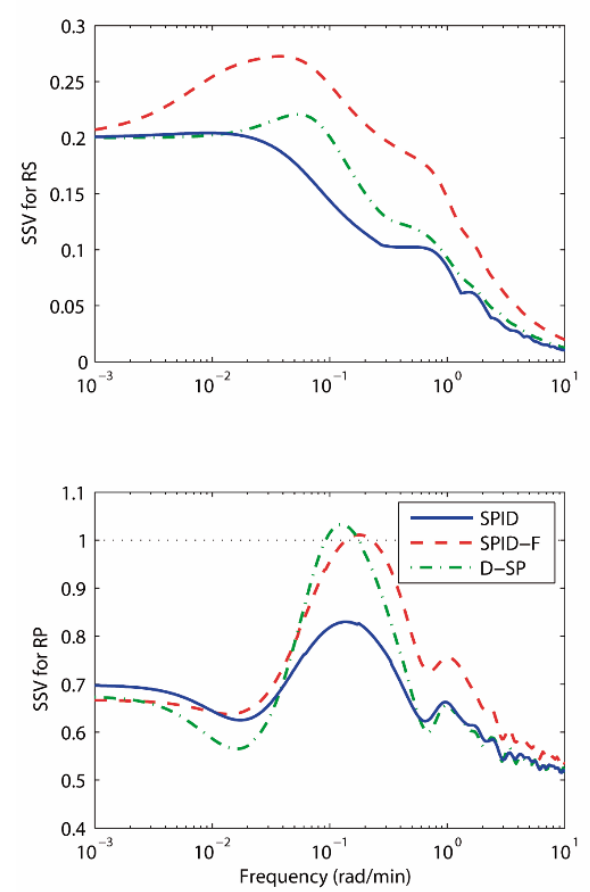

Figure 8. SSV for robust stability and robust performance in example 1.

\subsection{Example 2: $3 \times 3$ Tyreus distillation column}

The transfer matrix of this process is given by (52) in (Tyreus, 1979). Due to time delays, there are no realizable configurations according to section 2 . Therefore, it is necessary to add an additional block N(s) with delays. To obtain realizability by adding the minimum quantity of delays, the only choice is configuration $1-2-3$ with $n_{11}(\mathrm{~s})=e^{-0.09 \mathrm{~s}}, n_{22}(\mathrm{~s})=1$ and $n_{33}(\mathrm{~s})=e^{-0.26 \mathrm{~s}}$. Then, the proposed methodology is applied to the new augmented process $G(s) \cdot N(s)$ given by (53).

$$
\mathrm{G}_{\mathrm{T}}(\mathrm{s})=\left(\begin{array}{cccc}
\frac{1.986 e^{-0.71 s}}{66.7 s+1} & \frac{-5.24 e^{-60 s}}{400 s+1} & \frac{-5.984 e^{-2.24 s}}{14.29 s+1} \\
\frac{-0.0204 e^{-0.59 s}}{(7.14 s+1)^{2}} & \frac{0.33 e^{-0.68 s}}{(2.38 s+1)^{2}} & \frac{-2.38 e^{-0.42 s}}{(1.43 s+1)^{2}} \\
\frac{-0.374 e^{-7.75 s}}{22.22 s+1} & \frac{11.3 e^{-3.79 s}}{(21.74 s+1)^{2}} & \frac{9.811 e^{-1.59 s}}{11.36 s+1}
\end{array}\right)
$$




$$
\mathrm{G}_{\mathrm{T}}^{\mathrm{N}}(\mathrm{s})=\left(\begin{array}{cccc}
\frac{1.986 e^{-0.8 s}}{66.7 s+1} & \frac{-5.24 e^{-60 s}}{400 s+1} & \frac{-5.984 e^{-2.5 s}}{14.29 s+1} \\
\frac{-0.0204 e^{-0.68 s}}{(7.14 s+1)^{2}} & \frac{0.33 e^{-0.68 s}}{(2.38 s+1)^{2}} & \frac{-2.38 e^{-0.68 s}}{(1.43 s+1)^{2}} \\
\frac{-0.374 e^{-7.84 s}}{22.22 s+1} & \frac{11.3 e^{-3.79 s}}{(21.74 s+1)^{2}} & \frac{9.811 e^{-1.85 s}}{11.36 s+1}
\end{array}\right)
$$

The common output delays are obtained as $\theta_{1}=0.8 \mathrm{~min}, \theta_{2}=0.68 \mathrm{~min}$ and $\theta_{3}=1.85 \mathrm{~min}$. Then, the fast model $G_{o}(\mathrm{~s})$ is given by:

$$
\mathrm{G}_{o}(\mathrm{~s})=\left(\begin{array}{cccc}
\frac{1.986}{66.7 s+1} & \frac{-5.24 e^{-59.2 s}}{400 s+1} & \frac{-5.984 e^{-1.7 s}}{14.29 s+1} \\
\frac{-0.0204}{(7.14 s+1)^{2}} & \frac{0.33}{(2.38 s+1)^{2}} & \frac{-2.38}{(1.43 s+1)^{2}} \\
\frac{-0.374 e^{-5.99 s}}{22.22 s+1} & \frac{11.3 e^{-1.94 s}}{(21.74 s+1)^{2}} & \frac{9.811}{11.36 s+1}
\end{array}\right)
$$

The process elements of the first and third rows are FOPTD systems, and consequently, the controller parameters can be obtained using the expressions in (32) after specifying the desired closed-loop time constants $\lambda_{1}=17 \mathrm{~min}$ and $\lambda_{3}=21 \mathrm{~min}$. These values have been selected to achieve similar settling times than other authors. On the other hand, the system elements of the second row have relative degree equal to two and therefore, the case 2 of Table 1 can be applied. According to (26), $\lambda_{2}=24$ min and $\tau=6$ min are selected in order to achieve a critical damping response $(\xi=1)$ with a settling time similar to that of the other loops. The free-delay-open-loop transfer functions are as follows:

$$
l_{1}(s)=\frac{1}{17 s} \quad l_{2}(s)=\frac{1}{24 s(6 s+1)} \quad l_{3}(s)=\frac{1}{21 s}
$$

Assuming $G_{n}(s)=G_{T}^{N}(s)$ and after selecting the configuration 1-2-3, the controller elements of $\mathrm{Cd}(\mathrm{s})$ and $\mathrm{Co}(\mathrm{s})$ are obtained according to (18) without approximations, as follows: 


$$
\begin{gathered}
C d(s)=\left(\begin{array}{ccc}
\frac{(66.7 s+1)}{33.76 s} & 0 & 0 \\
0 & \frac{(2.38 \cdot s+1)^{2}}{7.92 \cdot(6 s+1) \cdot s} & 0 \\
0 & 0 & \frac{(11.36 s+1)}{206 s}
\end{array}\right) \\
C o(s)=\left(\begin{array}{ccc}
\frac{89.08 \cdot s \cdot e^{-592 s}}{(400 s+1)} & \frac{101.7 \cdot s e^{-1.7 s}}{(14.29 s+1)} \\
0 & 0 & \frac{57.12 \cdot s \cdot(6 s+1)}{(1.43 s+1)^{2}} \\
\frac{0.4896 \cdot s \cdot(6 s+1)}{(7.14 s+1)^{2}} & \frac{-237.3 \cdot s e^{-1.94 s}}{(21.74 s+1)^{2}} & 0
\end{array}\right)
\end{gathered}
$$

Although the process is a $3 \times 3$ system, the complexity of the elements of (56) and (57) is as simple as that of the elements obtained for $2 \times 2$ processes. The closed-loop system response is shown in Figure 9. There are unit step changes at $t=1 \mathrm{~min}$ in the first reference, at $t=333 \mathrm{~min}$ in the second one, and at $t=666 \mathrm{~min}$ in the third one. For comparison, other control methodologies are also shown: the pure centralized control of Wang in (Wang, 2003) and the analytical decoupling control of Liu in (Liu et al., 2007).

The proposed design achieves perfect decoupling performance without overshoot or inverse response in the outputs. It achieves similar IAE values than those of Liu's controller with a similar response. The Wang's controller obtains the worst IAE values, although it has the smaller TV values. These values are listed in Table 3. In this example the objective is to show that the proposed controller achieves a good compromise between complexity and performance. Note that it obtains similar response as Liu's controller but with simpler controller elements. Liu's controller elements are of high order, transfer functions of fourth, five or six order plus dead-time, while proposed controller uses only first or second order models, as shown in equations (56) and (57). In addition, the design procedures used in Liu's approach are more complex than that of the proposed one. 

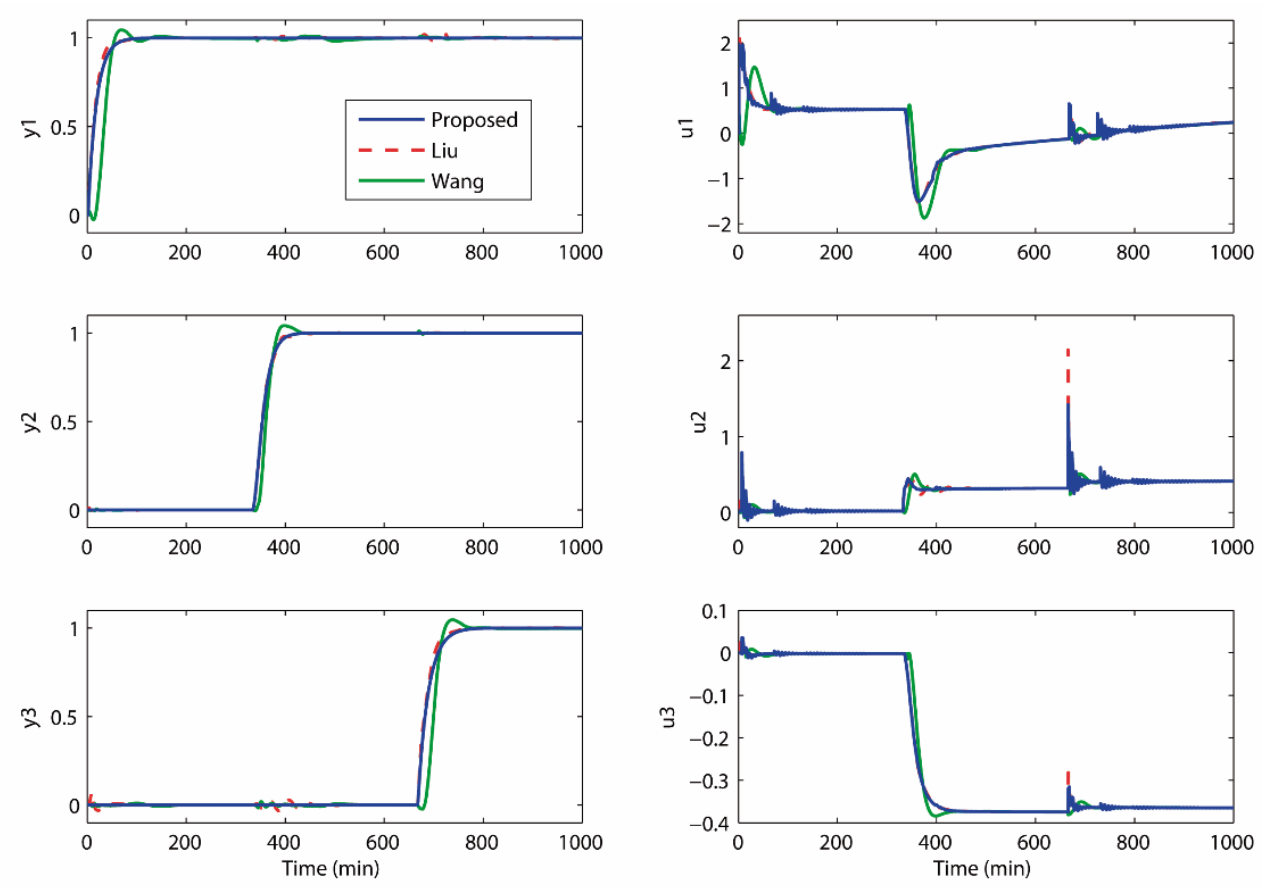

Figure 9. Outputs and control signals of the step response in example 2.

Table 3. Performance indices for each method in example 2.

\begin{tabular}{ccccccccc}
\hline Method & $\mathbf{I A E}_{\mathbf{1}}$ & $\mathbf{I A E}_{\mathbf{2}}$ & $\mathbf{I A E}_{\mathbf{3}}$ & $\mathbf{T V}_{\mathbf{1}}$ & $\mathbf{T V}_{\mathbf{2}}$ & $\mathbf{T V}_{\mathbf{3}}$ & $\boldsymbol{\mu}_{\mathrm{RS}}$ & $\boldsymbol{\mu}_{\mathrm{RP}}$ \\
\hline Proposed & 17.8 & 24.7 & 22.8 & 22.2 & 12.5 & 1.1 & 0.52 & 1.04 \\
Liu & 17.5 & 25.1 & 22.9 & 9.7 & 3.2 & 0.6 & 0.23 & 0.76 \\
Wang & 37.8 & 31 & 37.5 & 9.5 & 1.7 & 0.5 & 0.22 & 1.23 \\
\hline
\end{tabular}

Using the weights in (60), a $\mu$-analysis similar to that of the previous example is carried out to investigate the robustness. It shows that the proposed control and the Liu's controller have similar RS and RP around all frequencies; nevertheless, the proposed control shows some peaks at frequencies around $1 \mathrm{rad} / \mathrm{min}$ where the RP will deteriorate. The peak values are listed in Table 3. On the other hand, the Wang's controller does not fulfill RP condition (50) at low frequencies, where it has an important peak value.

$$
\begin{aligned}
& \mathrm{W}_{\mathrm{I}}(\mathrm{s})=\mathrm{W}_{\mathrm{I}}(\mathrm{s}) \cdot \mathrm{I}=\frac{(2 \mathrm{~s}+0.2)}{\mathrm{s}+1} \cdot \mathrm{I} \\
& \mathrm{W}_{\mathrm{P}}(\mathrm{s})=\mathrm{W}_{\mathrm{P}}(\mathrm{s}) \cdot \mathrm{I}=\frac{(\mathrm{s} / 2.75+0.012)}{\mathrm{s}} \cdot \mathrm{I}
\end{aligned}
$$



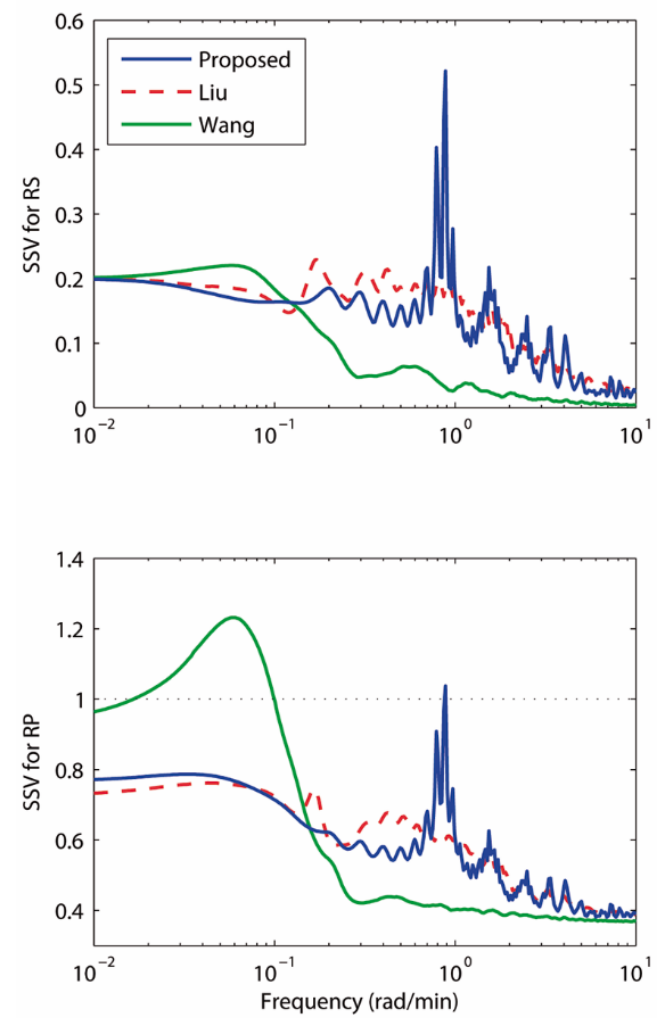

Figure 10. SSV for robust stability and robust performance in example 2.

\subsection{Example 3: Unstable process}

The transfer matrix of this $2 \times 2$ process is given by (59). It has important time delays and no multivariable RHP zeros. Nevertheless, its diagonal elements are unstable (Flesch et al., 2011).

$$
G_{U}(s)=\left(\begin{array}{cc}
\frac{-1.6 e^{-4 s}}{2.6 s-1} & \frac{0.6 e^{-6 s}}{2.5 s+1} \\
\frac{0.7 e^{-5 s}}{3 s+1} & \frac{-1.7 e^{-3 s}}{2.2 s-1}
\end{array}\right)
$$

As it was commented in section 2.5, in case of unstable poles, the implementation scheme in Figure 5 must be used to achieve internal stability. In addition, it is necessary to design a filter $\mathrm{F}(\mathrm{s})$ in order to cancel the unstable poles in $\mathrm{S}(\mathrm{s})$. This design is easier performed using a discrete representation of the plant. Using a sample time of $0.2 \mathrm{~s}$, the discrete plant model is given by 


$$
G_{U}(z)=\left(\begin{array}{cc}
\frac{-0.1279 z^{-20}}{z-1.08} & \frac{0.04797 z^{-30}}{z-0.92} \\
\frac{0.04515 z^{-25}}{z-0.9355} & \frac{-0.1618 z^{-15}}{z-1.095}
\end{array}\right)
$$

According to the conditions about time delays and unstable poles of section 2.2, configuration 1-2 must be selected for realizability. The common output delays are $\theta_{1}=4 \mathrm{~s}$ and $\theta_{2}=3 \mathrm{~s}$ (or 20 and 15 samples times, respectively). Therefore, the fast model $G_{o}$ (s) is given by:

$$
G_{o}(z)=\left(\begin{array}{cc}
\frac{-0.1279}{z-1.08} & \frac{0.04797 z^{-10}}{z-0.92} \\
\frac{0.04515 z^{-10}}{z-0.9355} & \frac{-0.1618}{z-1.095}
\end{array}\right)
$$

The two desired open-loop process $l_{i}(\mathrm{~s})$ can be specified according to the third case of Table 1. Using (28) and a critical damping factor as specification, parameters $\lambda_{1}=0.4461 \mathrm{~s}$ and $\lambda_{2}=0.3775 \mathrm{~s}$ are obtained. Then, the desired fast-open-loop processes are given by (62) and their discrete models by (63).

$$
\begin{aligned}
& l o_{1}(s)=\frac{s+1 / 2.6}{0.4461 \cdot s \cdot(s-1 / 2.6)} \quad l o_{2}(s)=\frac{s+1 / 2.2}{0.3775 \cdot s \cdot(s-1 / 2.2)} \\
& l o_{1}(z)=\frac{0.48372(z-0.9259)}{(z-1.08) \cdot(z-1)} \quad l o_{2}(z)=\frac{0.57946(z-0.913)}{(z-1.095) \cdot(z-1)}
\end{aligned}
$$

The controller elements are calculated by means of (14) using the continuous or discrete representation. The resultant discrete elements are:

$$
C d(z)=\left(\begin{array}{cc}
\frac{-3.643(z-0.9231)}{z-1} & 0 \\
0 & \frac{-3.4281(z-0.909)}{z-1}
\end{array}\right)_{(64)}
$$




$$
\operatorname{Co}(z)=\left(\begin{array}{cc}
0 & \frac{-0.0881 \cdot(z-1.081) \cdot(z-1) \cdot z^{-10}}{(z-0.9355) \cdot(z-0.9131)} \\
\frac{-0.1071 \cdot(z-1.068) \cdot(z-1) \cdot z^{-10}}{(z-0.926) \cdot(z-0.9231)} & 0
\end{array}\right)
$$

Since each closed-loop process has a dominant zero at $s=-1 / \mathrm{p}_{\mathrm{i}}$, a reference filter is used to avoid peaks in the responses. It is given by

$$
F f(z)=\left(\begin{array}{cc}
\frac{0.07404}{(z-0.9259)} & 0 \\
0 & \frac{0.0869}{(z-0.9131)}
\end{array}\right)
$$

In order to implement the scheme of Figure 5, block S(z) in (40) must be calculated and a stable diagonal filter $\mathrm{F}(\mathrm{z})$ must be designed to eliminate the unstable poles in $\mathrm{S}(\mathrm{z})$ and achieve internal stability. Using (41), the elements of $\mathrm{F}(\mathrm{z})$ are designed to cancel the unstable pole at $\mathrm{z}=1.08$ in the first element of $\mathrm{S}(\mathrm{s})$, and at $\mathrm{z}=1.095$, in the last one, respectively. Moreover, a closed-loop pole in $\mathrm{z}=0.9$ and $\mathrm{z}=0.95$ are respectively used to define the closedloop disturbance rejection response. The filter matrix is given by

$$
F(z)=\left(\begin{array}{cc}
\frac{9.232 \cdot(z-0.9892)}{(z-0.9)} & 0 \\
0 & \frac{5.439 \cdot(z-0.9908)}{(z-0.95)}
\end{array}\right)
$$

The closed-loop system response of the proposed method is shown in Figure 11. There are unit step changes at $t=5 \mathrm{~s}$ in the first reference, at $t=40 \mathrm{~s}$ in the second one, and a 0.05 step in both process inputs as load disturbances at $t=80 \mathrm{~s}$. For comparison, the multivariable filtered Smith Predictor (MIMO-FSP) in (Flesch et al., 2011) is also presented. The IAE values are listed in Table 4. The proposed design achieves similar performance than that in (Flesch et al., 2011), with a decoupling response, bit smaller settling times and smaller IAE and TV values. These results are expected as the proposed strategy has improved the primary 
controller tuning of the dead-time compensator structure. It is important to emphasize here that in the MIMO-FSP, the focus was the predictor structure and not the primary controller tuning. Therefore, the interesting result of this example is that using a more elaborated primary controller design with decoupling objectives, it is possible to improve the responses of the MIMO-FSP. From Table 4, it can be noticed that the TV and IAE values of the MIMOFSP are respectively 50\% and 10\% higher than the ones in the proposed controller.

Table 4. Performance indices for each method in example 3.

\begin{tabular}{ccccc} 
& \multicolumn{2}{c}{ Proposed } & \multicolumn{2}{c}{ F-SP } \\
\cline { 2 - 5 } IAE & 7.6 & 6.7 & 8.4 & 8.2 \\
TV & 0.02 & 0.02 & 0.03 & 0.03
\end{tabular}
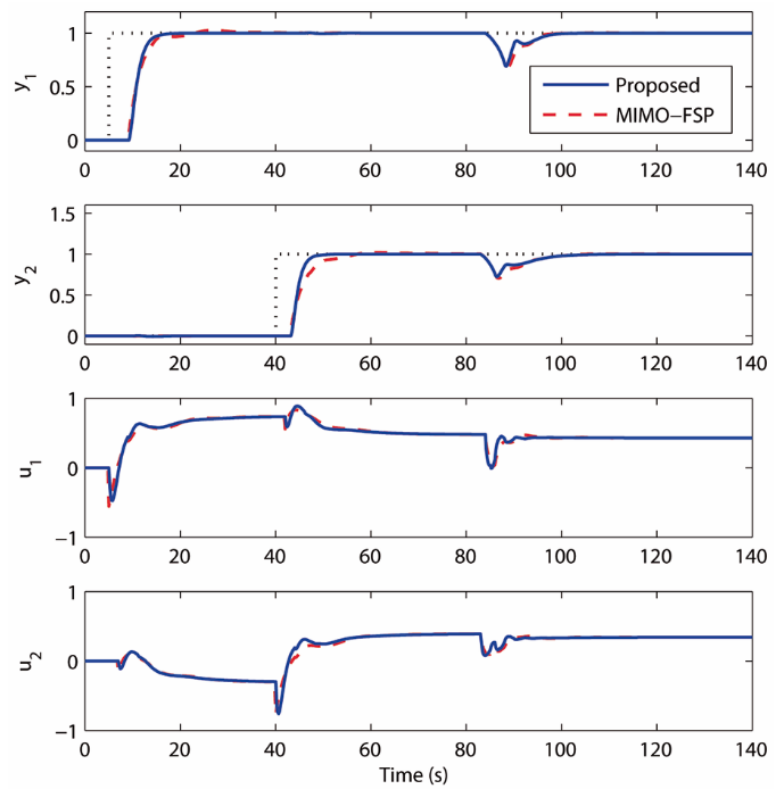

Figure 11. Outputs and control signals of the step response in example 3.

\section{Conclusions}

In this work, a new methodology of decoupling Smith predictor for multivariable square processes with multiple time delays has been proposed. It is based on the structure of centralized inverted decoupling control, which is combined with the SP structure. From a 
compact matrix formulation, the generalized expressions for $n \times n$ processes have been obtained. The realizability conditions for applying the method were provided, even for unstable processes. Furthermore, for processes with all of its elements as stable FOPTD systems, PID controllers and filtered derivative compensators plus time delays are obtained, which can be easily implemented in commercial distributed control systems. Expressions for direct calculations in this case have been provided. The method has been illustrated through three simulation examples, and comparisons with other works have demonstrated that the proposed methodology achieves similar or better performance.

The main advantages of the proposed method over other methodologies are the following:

- The simplicity of the controller elements. They do not contain sum of transfer functions and have similar or smaller order than that of the process transfer functions. Using other methods (conventional decoupling schemes or more advanced techniques), in some cases it is possible to have non-rational or complicated controller elements which are difficult to implement.

- Easiness for specifying closed-loop performance requirements as simple time response specifications.

- The complexity of the controller elements is independent of the system size, which is a great advantage over other multivariable methodologies.

- Using the proposed diagonal filter, the disturbance rejection response can be improved without modifying the nominal reference tracking response.

- This filter can also be used to obtain a stable implementation of the controller in the case of unstable systems. 
On the other hand, the main drawback of the proposed methodology is that it cannot be applied to some processes with multivariable RHP zeros because the inverted decoupling structure results internally unstable. Consequently, further work must be performed to extend this method to these cases. Another future work is the application of the proposed strategy in an experimental setup.

\section{Acknowledgements}

This work was supported by the Autonomous Government of Andalusia (Spain) under the Excellence Project P10-TEP-6056; and the Spanish Ministry of Economy and Competitiveness under Grant DPI2012-37580-C02-01. Julio E. Normey-Rico thanks CNPq for financial support.

\section{References}

Bresch-Pietri, D., Chauvin, J., \& Petit, N. (2012). Adaptive control scheme for uncertain time-delay systems. Automatica, 48(8), 1536-1552.

Chen, J., He, Z.-F., \& Qi, X. (2011). A new control method for MIMO first order time delay non-square systems. J. Process Control, 21(4), 538-546.

Flesch, R. C. C., Torrico, B. C., Normey-Rico, J. E., \& Cavalcante, M. U. (2011). Unified approach for minimal output dead time compensation in MIMO processes. J. Process Control, 21(7), 1080-1091. doi: 10.1016/j.jprocont.2011.05.005

Galdos, G., Karimi, A., \& Longchamp, R. (2010). $\mathrm{H}_{\infty}$ Controller design for spectral MIMO models by convex optimization. J. Process Control, 20(10), 1175-1182.

García, P., \& Albertos, P. (2010). Dead-time-compensator for unstable MIMO systems with multiple time delays. J. Process Control, 20(7), 877-884.

Garrido, J., Vázquez, F., \& Morilla, F. (2011). An extended approach of inverted decoupling. J. Process Control, 21(1), 55-68.

Garrido, J., Vázquez, F., \& Morilla, F. (2013). Centralized Inverted Decoupling Control. Ind. Eng. Chem. Res., 52(23), 7854-7866.

Garrido, J., Vázquez, F., \& Morilla, F. (2014a). Inverted decoupling internal model control for square stable multivariable time delay systems. J. Process Control, 24(11), 1710-1719.

Garrido, J., Vázquez, F., \& Morilla, F. (2014b). Multivariable PID control by decoupling. International Journal of Systems Science. doi: 10.1080/00207721.2014.911390

Garrido, J., Vázquez, F., \& Morilla, F. (2014c). Smith predictor with inverted decoupling for stable TITO processes with time delays. Paper presented at the Proceedings of 19th IEEE International Conference on Emerging Technologies and Factory Automation, Barcelona (Spain).

He, M.-J., Cai, W.-J., \& Wu, B.-F. (2006). Design of decentralized IMC-PID controller based on dRI analysis. AIChE J., 52(11), 3852-3863.

Huang, H. P., Jeng, J. C., Chiang, C. H., \& Pan, W. (2003). A direct method for multi-loop PI/PID controller design. J. Process Control, 13(8), 769-786.

Jerome, N. F., \& Ray, W. H. (1986). High-performance multivariable control strategies for systems having time delays. AIChE J., 32(6), 914-931. doi: 10.1002/aic.690320603

Krstic, M. (2010). Lyapunov stability of linear predictor feedback for time-varying delay processes. IEEE Trans. Automat. Control, 55(2), 554-559.

Liu, T., Zhang, W., \& Gao, F. (2007). Analytical decoupling control strategy using a unity feedback control structure for MIMO processes with time delays. J. Process Control, 17(2), 173-186. doi: 10.1016/j.jprocont.2006.08.010

Mirkin, L., Palmor, Z., \& Shneiderman, D. (2011). Dead-time compensation for systems with multiple I/O delays: A loop-shifting approach. IEEE trans Automat Control, 56(11), 2542-2554. 
Morilla, F., Garrido, J., \& Vazquez, F. (2013). Multivariable Control by Decoupling. Revista Iberoamericana De Automatica E Informatica Industrial, 10(1), 3-17. doi: 10.1016/j.riai.2012.11.001

Normey, J. E., \& Camacho, E. F. (2008). Dead-time compenstators: A survey. Control Eng. Pract., 16(4), 407428.

Normey-Rico, J. E., \& Camacho, E. F. (2006). Predicción para control: Una panorámica del control de procesos con retardo. Rev. Iberoam. Autom. Inform. Ind., 3(4), 5-25.

Normey-Rico, J. E., \& Camacho, E. F. (2007). Control of dead-time processes: Springer-Verlag.

Normey-Rico, J. E., \& Camacho, E. F. (2009). Unified approach for robust dead-time comensator design. J. Process Control, 19(1), 38-47.

Palmor, Z. J. (1996). Time delay compensation: Smith predictor and its modifications The Control Hand Book (pp. 224-237): CRC Press and IEEE Press.

Rao, A. S., \& Chindambaram, M. (2006). Smith delay compensator for multivariable non-square systems with multiple time delays. Comput. Chem. Eng., 30(8), 1243-1255.

Rao, A. S., Rao, V. S., \& Chidambaram, M. (2007). Delay-compensated controllers for two-input/two-output (TITO) multivariable processes. Asia-Pac. J. Chem. Eng., 2, 510-516.

Santos, T. L. M., Flesch, R. C. C., \& Normey-Rico, J. E. (2014). On the filtered Smith predictor for MIMO processes with multiple time delays. J. Process Control, 24(4), 383-400.

Skogestad, S., \& Postlethwaite, I. (2005). Multivariable Feedback Control: Analysis and Design (2 ed.). Chichester, England: John Wiley \& sons.

Smith, O. J. M. (1957). Closed control of loops with dead time. Chem. Eng. Prog., 53, 217-219.

Sánchez-Peña, R. S., Bolea, Y., \& Puig, V. (2009). MIMO smith predictor: global and structured robust performance analysis. J. Process Control, 19(1), 163-177. doi: 10.1016/j.jprocont.2007.12.004

Tyreus, B. D. (1979). Multivariable control system design for an industrial distillation column. Ind. Eng. Chem. Process Des. Dev., 18(1), 177-182. doi: 10.1021/i260069a025

Vijay-Kumar, V., Rao, V. S. R., \& Chidambaram, M. (2012). Centralized PI controllers for interacting multivariable processesby synthesis method. ISA Trans., 51(3), 400-409.

Vázquez, F., Morilla, F., \& Dormido, S. (1999). An iterative method for tuning decentralized PID controllers. Paper presented at the Proceedings of the 14th IFAC World Congress, Beijing, China.

Wang, Q. G. (2003). Decoupling Control. Berlin-Heidelberg: Springer-Verlag.

Wang, Q. G., Zhang, Y., \& Chiu, M. S. (2002). Decoupling internal model control for multivariable systems with multiple time delays. Chem. Eng. Sci., 57(1), 115-124.

Wang, Q. G., Zhang, Y., \& Chiu, M. S. (2003). Non-interacting control design for multivariable industrial processes. J. Process Control, 13(3), 253-265. doi: 10.1016/S0959-1524(02)00028-8

Wang, Q. G., Zou, B., \& Zhang, Y. (2000). Decoupling Smith predictor design for multivariable systems with multiple time delays. Chem. Eng. Res. Des., 78(4), 152-170.

Xie, L. B., Shiehb, L. S., Pana, F., Tsaic, J. S. H., \& Canelond, J. I. (2014). Design of decoupling and tracking controllers for continuous-time transfer function matrices with multiple time delays. J. Process Control, 24(1), 152-170.

Xiong, Q., Cai, W. J., \& He, M. J. (2007). Equivalent transfer function method for PI/PID controller design of MIMO processes. J. Process Control, 17(8), 665-673.

Zhang, H., Shi, Y., \& Wang, J. (2014). On Energy-to-Peak Filtering for Nonuniformly Sampled Nonlinear Systems: A Markovian Jump System Approach. IEEE Trans. on Fuzzy Systems, 22(1), 212-222.

Zhang, H., \& Wang, J. (2015). State Estimation of Discrete-tiem Takagi-Sugeno Fuzzy Systems in a Network Environment. IEEE Trans. on Cybernetics, In press, 12. doi: 10.1109/TCYB.2014.2354431 